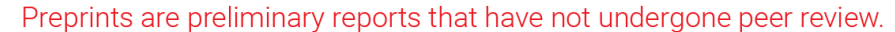 They should not be considered conclusive, used to inform clinical practice, or referenced by the media as validated information. \\ Tauber antegrade sclerotherapy: the importance of phlebography in choosing the correct vein.
}

\section{Edoardo Guida}

Department of Paediatric Surgery and Urology, Institute for Maternal and Child Health - IRCCS "Burlo Garofolo", 34137 Trieste, Italy

\section{Alessandro Boscarelli ( $\square$ tboscar@hotmail.it)}

Department of Paediatric Surgery and Urology, Institute for Maternal and Child Health - IRCCS "Burlo Garofolo", 34137 Trieste, Italy https://orcid.org/0000-0003-1934-1764

\section{Miriam Duci}

Pediatric Surgery Division, Women's and Children's Health Dept, University of Padua, Padua, Italy.

\section{Giulia Ceschiutti}

University of Trieste, Faculty of Medicine and Surgery, 34127 Trieste, Italy

\section{Jurgen Schleef}

Department of Paediatric Surgery and Urology, Institute for Maternal and Child Health - IRCCS "Burlo Garofolo", 34137 Trieste, Italy

\section{Short Report}

Keywords: Varicocele, Tauber, Phlebography, Complications, Children

Posted Date: July 16th, 2021

DOI: https://doi.org/10.21203/rs.3.rs-715962/v1

License: (c) (i) This work is licensed under a Creative Commons Attribution 4.0 International License. Read Full License 


\section{Abstract}

Tauber antegrade sclerotherapy can be considered an established procedure for the treatment of varicocele in pediatric age. Herein, we share our experience with this technique and its tricks. The choice of the spermatic vein should be very accurate during the Tauber procedure, and a correct intraoperative phlebography is mandatory to avoid complications.

\section{Main Text}

The antegrade scrotal sclerotherapy is a combined radiological-surgical approach introduced by Tauber in 1988 [1]. To date Tauber antegrade sclerotherapy can be considered a valuable procedure for the treatment of varicocele in the pediatric population. Nonethless, some severe complications related to this procedure have been described in the literature, including sterile epididymitis (caused by the perivascular application of the sclerosing agent), testicular atrophy (due to testicular artery sclerotherapy), abdominal wall necrosis (incorrect sclerotherapy of the cremasteric vessels) and intestinal segmental necrosis (determined by accidental sclerotherapy of the internal iliac veins) $[2,3]$.

We retrospectively collected data of pediatric patients undergoing surgery for left varicocele at our Pediatric Surgery and Urology Department. The data extracted from the records were age, time of surgery, and complications. Continuous variables are reported as medians and interquartile ranges (IQR) and categorical variables as numbers and percentages.

A total of 88 male patients underwent Tauber procedure from July 2013 to May 2021. Median age was 15 years (range 10-20). Mean operative time was 40 minutes (range 25-120). Recurrence occurred in 4 patients $(4,5 \%)$, and a redo Tauber procedure was successfully performed. In two patients $(2,3 \%)$ antegrade scrotal sclerotherapy could not be achieved, and the procedure was converted to a retroperitoneal approach (Palomo). Notably, in two other patients $(2,3 \%)$, during Phlebography a vascular anomaly was noted. In the first case, we identified the left spermatic vein during the contrast medium injection into a vein of the deep pampiniform plexus but we noted a collateral vein starting from the proximal tract of the spermatic vein and headed towards the left colic space (Figure 1, left). As we could not predict the collateral vein's course, we decided to stop the Tauber procedure and selectively ligate the veins. In a clinical and ultrasound follow-up after 6 -months, no residual left varicocele was observed. In the second case, during the contrast medium injection into a vein of the superficial pampiniform plexus, the cremasteric area was identified at the lower abdominal wall level (Figure 1, right). Consequently, we decided to ligate the vein and choose a deeper vein of the plexus and the Tauber procedure was completed. No residual left varicocele was observed at clinical and ultrasound follow-up 6-months after surgery.

Concerning the last two complications described, it is worth highlighting two key-points: 1) The vein's correct choice should be very accurate during the Tauber procedure. The plexus' superficial vessels may often flow into the cremasteric area that leads blood to the inferior abdominal wall. Besides, it may be 
more challenging to cannulate a superficial vein than a deep plexus vein for the lower dimensions, resulting in bleeding or hematoma of the perivasal tissue, and, subsequently, choosing another vein may not be so simple; 2 ) although many researchers $[3,4]$ suggested performing a preoperative phlebography before proceeding with sclerotherapy, some authors described few false negatives even with a nonidentification of the presence of abnormal venous communications with the pelvic venous plexus and with serious complications such as ischemic necrosis of the sigmoid colon and lower abdominal wall necrosis [4]. However, we believe that a correct intraoperative phlebography should always be mandatory to avoid complications. Furthermore, three notions should be keept in mind to perform the right phlebography in order to reduce the risk of false negatives: a) The left spermatic vein should always be followed up to the intersection with the left renal vein to identify some very high collateral veins, such as internal iliac afferent veins, which may not be detected during incomplete phlebography; b) The patient's position on the operating table should be carefully considered to perform right phlebography up to the left renal vein, especially in the case of a tall boy. The operating table should always be positioned to the length lower limit to allow the correct access of phlebography's tool under the table, enabling the kidney lodge visualization. The visualization of a tall patient's renal lodge may not be as visible if the operating table has a single lower position. So, it may be useful to employ a table's lower extension to locate the child's legs, allowing the tool's correct location; c) The patient should maintain the Valsalva maneuver during phlebography and the sclerosing agent injection. The Valsalva maneuver could make visible the inversion of the venous flow through the small veins in the internal iliac veins, thus preventing one of the most severe complications related to this procedure [5].

In conclusion, although Tauber antegrade sclerotherapy can be considered a safe and effective procedure in the treatment of varicocele in children, some challenging complications could be avoided through small but not negligible technical details.

\section{References}

1. Tauber R, Pfeiffer D. Surgical atlas varicocele: Antegrade scrotal sclerotherapy. BJU Int. 2006 Dec;98(6):1333-44.

2. Fulcoli V, Costa G, Gigli F, Laurini L. Ischemic necrosis of the sigmoid colon after antegrade sclerotherapy of idiopathic varicocele: a case report. Urologia. 2013 Apr-Jun;80(2):162-4.

3. Boscolo-Berto R, Macchi V, Porzionato A, Morra A, Vezzaro R, Loukas M, Tubbs RS, De Caro R. Ischemic colitis following left antegrade sclerotherapy for idiopathic varicocele. Clin Anat. 2018 Sep;31(6):774-781.

4. Salerno S, Cannizzaro F, Lo Casto A, Romano P, Bentivegna E, Lagalla R. Anastomosi tra le vene spermatica interna sinistra e splancniche. Analisi retrospettiva su 305 pazienti [Anastomosis between the left internal spermatic and splanchnic veins. Retrospective analysis of 305 patients]. Radiol Med. 2000 May;99(5):347-51. Italian. 
5. Vicini P, Di Pierro GB, Grande P, Voria G, Antonini G, De Marco F, Di Nicola S, Gentile V. Large bowel infarct following antegrade scrotal sclerotherapy for varicocele: A case report. Can Urol Assoc J. 2014 Sep;8(9-10):E641-3.

\section{Declarations}

Funding Source: This article did not receive any specific grant from funding agencies in the public, commercial, or not-for-profit sectors.

Conflict of Interest Statement. The authors have no conflicts of interest related to this article to declare.

Availability of data and material: The material that support the findings of this study are available on request from the corresponding author.

Ethical approval: All procedures performed were in accordance with the ethical standards.

Consent to publish form: Patients/Legal Guardians/Study participants gave their consent to publish the data enclosed in this paper.

Authors' contributions: Dr Guida contributed to conception of the study and draft the initial manuscript; $\mathrm{Dr}$ Duci, Dr Boscarelli, and Dr Ceschiutti contributed to the acquisition of data and images; Dr Boscarelli revised the final manuscript; Dr Schleef supervised and revised the final manuscript. All the authors approved the final version of the manuscript and attest that they meet the ICMJE criteria for authorship.

Statement of ethics approval: The studies involving human participants were reviewed and approved by Health Care Ethnics Committee of IRCCS Burlo Garofolo of Trieste. Written informed consent was obtained from the individuals/legal guardians for the publication of any potentially identifiable images or data included in this article.

\section{Figures}




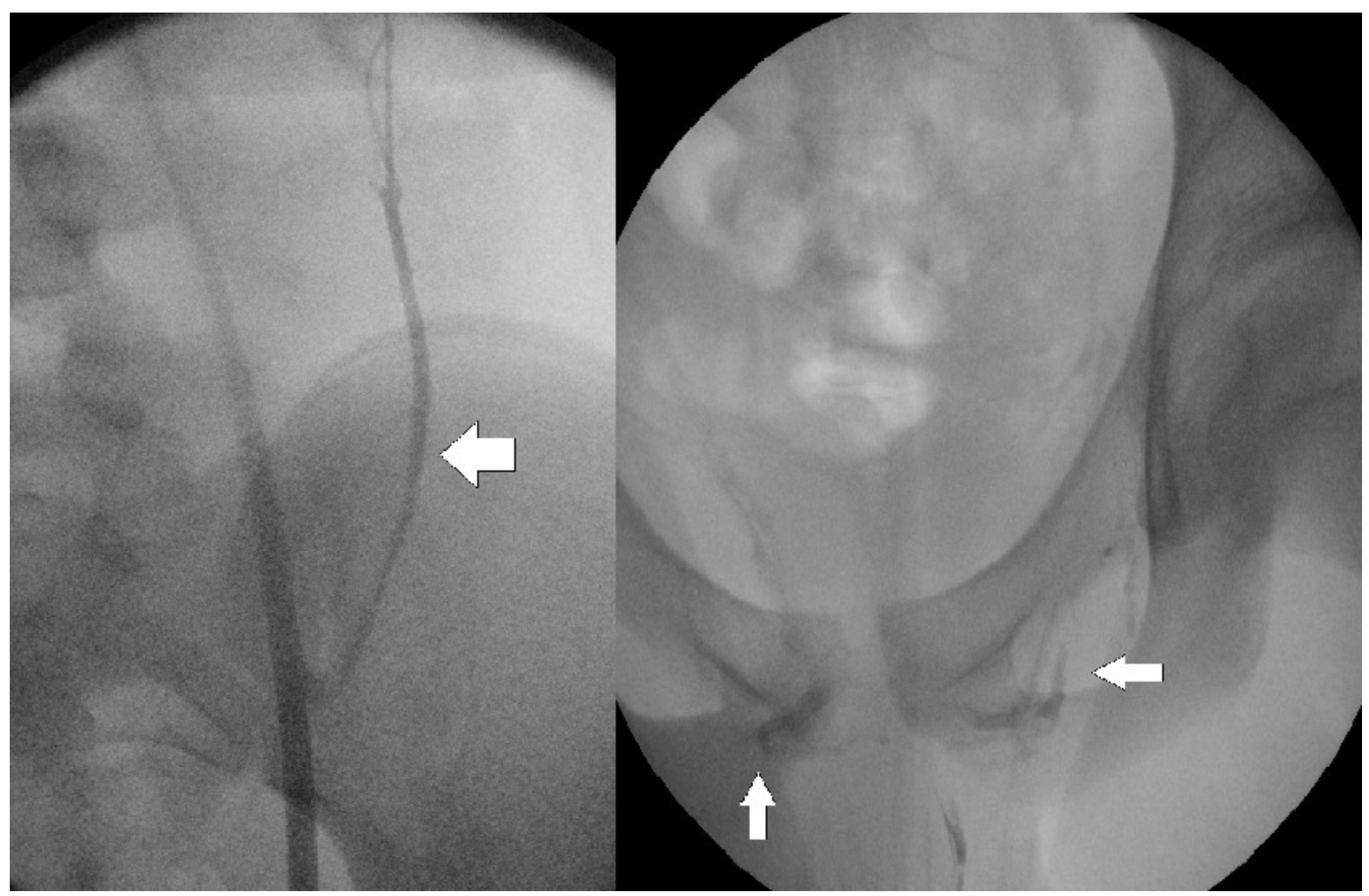

\section{Figure 1}

Intraoperative fluoroscopy showing a collateral vein which runs laterally in the left colic space (left), and fluoroscopy showing collateral circulation through the cremasteric area, after the contrast is injected into a vein of the superficial pampiniform plexus (right). 